Check for updates

Cite this: RSC Adv., 2017, 7, 44665

Received 23rd August 2017

Accepted 13th September 2017

DOI: $10.1039 / \mathrm{c} 7 \mathrm{ra0} 0326 \mathrm{~h}$

rsc.li/rsc-advances

\section{Separation performance of a large $\pi$-conjugated truxene-based dendrimer as stationary phase for gas chromatography}

\author{
Meng Wang, Yinhui Yang, Meiling Qi (D) and Jinliang Wang*
}

This work describes the separation performance of the thiophene-functionalized truxene dendrimer (TFTD) as the stationary phase for gas chromatography (GC). An investigation was performed regarding its polarity, column efficiency, separation performance, column repeatability, reproducibility and thermal stability. As a result, the TFTD column showed a high column efficiency of 4420 plates $\mathrm{m}^{-1}$, and weak polarity on the basis of its McReynolds constants. It exhibited high resolving capability for a wide range of analytes, particularly for phenols, anilines, alkylated and halogenated benzenes, showing distinct advantages over the TFT and commercial columns. Moreover, the TFTD column displayed good column repeatability and reproducibility with RSD values in the range of $0.02-0.19 \%$ for intra-day, $0.72-1.7 \%$ for inter-day and $1.3-4.9 \%$ for between-column, respectively. The present work demonstrates the promising future of $\pi$ conjugated thiophene-functionalized dendrimers in separation science.

\section{Introduction}

Dendrimers have a well-defined dendritic molecular shape with a porous architecture and favorable physicochemical properties, and have gained increasing attention in the areas of molecular electronics and sensing,,${ }^{1,2}$ drug delivery, ${ }^{3}$ membrane separation ${ }^{4,5}$ and surface functionalization on nanoparticles. ${ }^{6}$ In separation science, dendrimers such as 1,4-butanedioldiglycidyl ether (BDDE), poly(amidoamine) and carbosilane have also found their use either as the stationary phases for ion chromatography, ${ }^{7,8}$ high performance liquid chromatography ${ }^{9}$ and capillary electrochromatography ${ }^{10}$ or as the sorbents for solid phase extraction ${ }^{11}$ and solid phase microextraction..$^{12}$ To date, however, no reports are available on utilizing dendrimers as stationary phases for capillary gas chromatography (GC).

Thiophene-functionalized truxene (TFT, Fig. 1) derivatives, especially TFT-based dendrimers (TFTD), are promising organic field-effect transistors and light harvesting materials due to their unique large $\pi$-conjugated architecture, outstanding optoelectronic properties, good solubility and high chemical/ thermal stability. ${ }^{13-16}$ In our previous work, ${ }^{17}$ the TFT stationary phase showed good separation performance as GC stationary phase for halogenenated and alkyl benzene analytes and di-/tri-chlorobiphenyls. The TFTDs that were constructed through joining three TFT units onto the TFT core by oligo(thienylethynylene)s in a dendritic architecture ${ }^{14,15}$ have a largely

Key Laboratory of Cluster Science, Ministry of Education of China, Beijing Key Laboratory of Photoelectronic/Electrophotonic Conversion Materials, School of Chemistry and Chemical Engineering, Beijing Institute of Technology, Beijing, 100081, China.E-mail: mlqi@bit.edu.cn; jinlwang@bit.edu.cn extended $\pi$-conjugated system compared to a single TFT building unit, and possess a well-defined porous structure between the adjacent rigid branches, which provide access to specific compound molecules. These advantageous features of TFTDs over TFT suggested their good potential as GC stationary

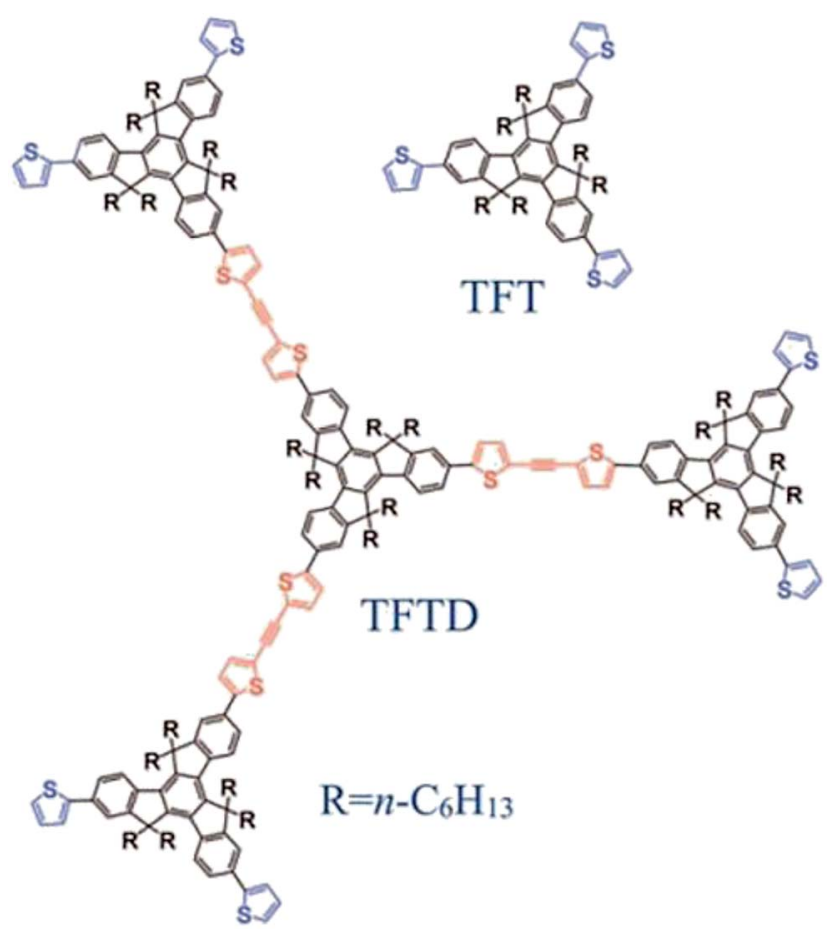

Fig. 1 The structures of TFTD and TFT. 
phases. Undoubtedly, their potential in this respect is quite worth exploration.

This work presents the investigation on the separation performance of one TFTD material (Fig. 1) as GC stationary phase. To our knowledge, this is the first example of utilizing large $\pi$-conjugated dendrimers in the field of chromatography. In this work, after statically coated onto a capillary column, the TFTD stationary phase was investigated in terms of column efficiency, polarity, separation performance and retention behaviors, column repeatability, reproducibility and thermal stability. A wide range of analytes and mixtures were utilized to evaluate its separation capability and retention behaviors utilizing the TFT column and commercial HP-5MS column as reference columns. Furthermore, the TFTD column was applied to the determination of isomer impurities in real samples.

\section{Experimental}

\subsection{Materials and equipment}

The TFTD and TFT stationary phases were synthesized following the method in our previous report. ${ }^{15}$ All the analytes in this work were of analytical grade. Bare fused-silica capillary tubing (0.25 mm i.d.) was purchased from Yongnian Optic Fiber Plant (Hebei, China). One HP-5MS capillary column $(5 \mathrm{~m} \times 0.25 \mathrm{~mm}$, i.d., film thickness $0.25 \mu \mathrm{m}$ ) coated with $5 \%$ phenylmethylpolysiloxane was purchased from Agilent Technologies. An Agilent 7890A gas chromatograph equipped with a split/ splitless injector, a flame ionization detector (FID) and an autosampler was employed for the GC separations. The carrier gas was high-purity nitrogen (99.999\%) and split injection was set at the split ratio $50: 1$. The temperatures at the injection port and the detector were $250{ }^{\circ} \mathrm{C}$ and $300{ }^{\circ} \mathrm{C}$, respectively. Thermogravimetric analysis (TGA) was performed on a Q500 Thermogravimetric Analyzer from TA Instruments Inc. (Delaware, USA) from $30{ }^{\circ} \mathrm{C}$ to $700{ }^{\circ} \mathrm{C}$ at a ramp rate of $10{ }^{\circ} \mathrm{C} \mathrm{min}{ }^{-1}$ under nitrogen.

\subsection{Fabrication of the TFTD and TFT capillary columns}

Prior to coating, a fused-silica capillary column $(5 \mathrm{~m} \times 0.25$ $\mathrm{mm}$, i.d.) was pretreated with a saturated methanol solution of sodium chloride to roughen its inner wall. Then, the column was conditioned up to $200{ }^{\circ} \mathrm{C}$ and held for $3 \mathrm{~h}$ with the purging of nitrogen. Afterwards, the capillary column was statically coated with the solution of the TFTD stationary phase in dichloromethane $(0.08 \%, \mathrm{w} / \mathrm{v})$ at $40{ }^{\circ} \mathrm{C}$. After the column was filled with the solution, its one end was sealed up and the other end was connected to a vacuum system to evaporate the solvent. Finally, the coated capillary column was conditioned from $40{ }^{\circ} \mathrm{C}$ to $180{ }^{\circ} \mathrm{C}$ at $1{ }^{\circ} \mathrm{C} \min ^{-1}$ and held for $7 \mathrm{~h}$ under nitrogen. Meanwhile, the TFT column was fabricated by the same procedure.

\section{Results and discussion}

\subsection{Inherent thermal stability, column efficiency and polarity}

The inherent thermal stability of the TFTD stationary phase was examined by TGA analysis prior to its coating onto a capillary column. As shown in Fig. $2 \mathrm{a}$, it is thermally stable up to $416{ }^{\circ} \mathrm{C}$ where about $5 \%$ weight loss occurs, suggesting its good thermal stability and feasibility as the stationary phase for GC separations. For the fabricated TFTD and TFT columns, their Golay curves, relating the height equivalent to a theoretical plate (HETP) to varying flow rate of carrier gas, were measured by $n$ dodecane at $120{ }^{\circ} \mathrm{C}$. As shown in Fig. 2, the TFTD column possessed a Golay curve comparable to the TFT column and showed the minimum HETP of $0.226 \mathrm{~mm}$, corresponding to the column efficiency of 4420 plates $\mathrm{m}^{-1}$. Moreover, the coating

Table 1 McReynolds constants of the TFTD, TFT and HP-5MS stationary phases ${ }^{a}$

\begin{tabular}{lcllllll}
\hline $\begin{array}{l}\text { Stationary } \\
\text { phase }\end{array}$ & $\mathrm{X}^{\prime}$ & $\mathrm{Y}^{\prime}$ & $\mathrm{Z}^{\prime}$ & $\mathrm{U}^{\prime}$ & $\mathrm{S}^{\prime}$ & $\begin{array}{l}\text { General } \\
\text { polarity }\end{array}$ & $\begin{array}{l}\text { Average } \\
\text { polarity }\end{array}$ \\
\hline TFTD & 68 & 110 & 83 & 155 & 148 & 564 & 113 \\
TFT & 78 & 143 & 125 & 78 & 155 & 579 & 116 \\
HP-5MS & 30 & 72 & 62 & 96 & 65 & 325 & 65
\end{tabular}

${ }^{a} \mathrm{X}^{\prime}$, benzene; $\mathrm{Y}^{\prime}$, 1-butanol; $\mathrm{Z}^{\prime}$, 2-pentanone; $\mathrm{U}^{\prime}$, 1-nitropropane; $\mathrm{S}^{\prime}$, pyridine. Temperature: $120^{\circ} \mathrm{C}$.
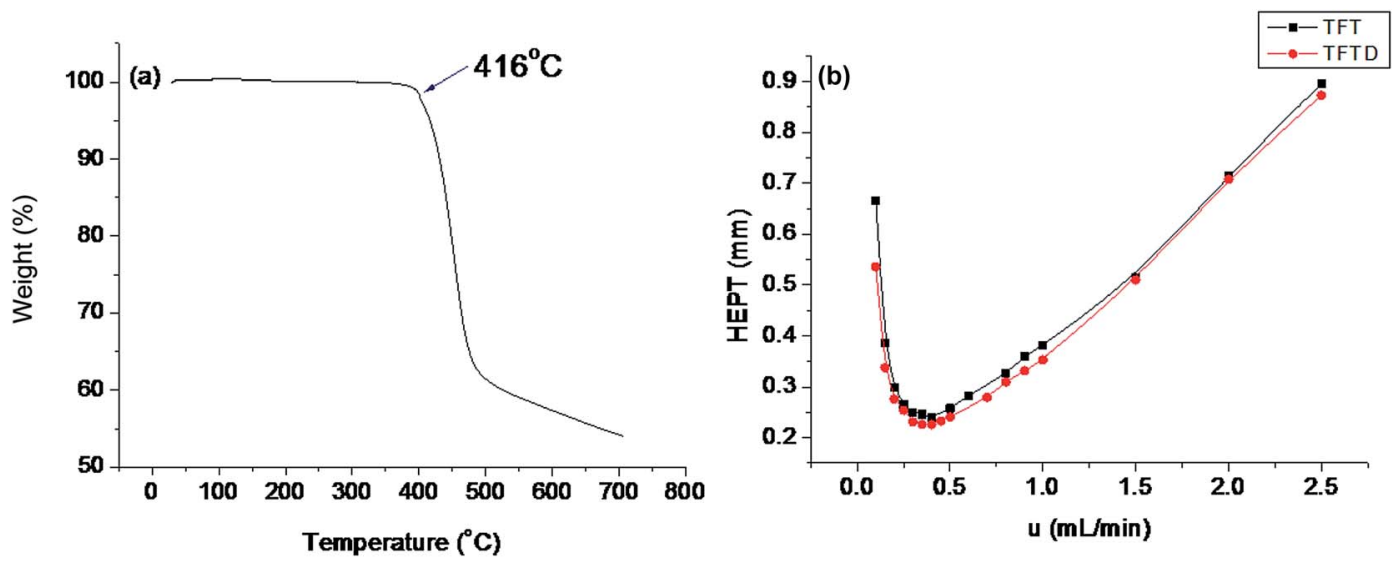

Fig. 2 (a) TGA curve of the TFTD stationary phase under nitrogen atmosphere and (b) Golay curves of the TFTD and TFT capillary columns determined by $n$-dodecane at $120^{\circ} \mathrm{C}$. 

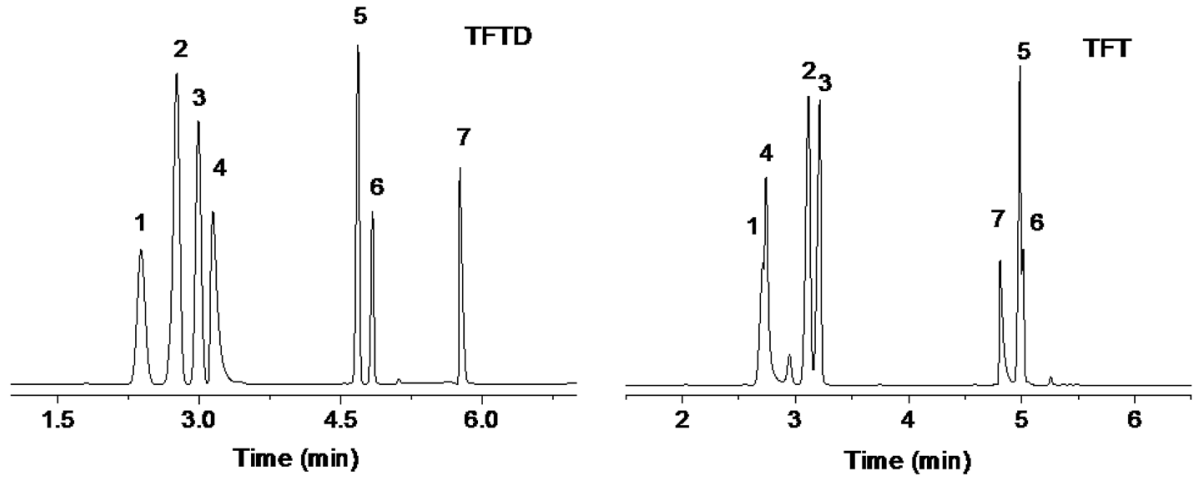

Fig. 3 GC separations of the mixture of 7 analytes on the TFTD column in comparison to the TFT column. Peaks: (1) $m$-cymene, (2) $n$-butylbenzene, (3) 1,2-dichlorobenzene, (4) 4-fluoroaniline, (5) $n$-hexylbenzene, (6) 1,2,3-trichlorobenzene, (7) 4-chlorophenol. Temperature program: $50{ }^{\circ} \mathrm{C}$ to $80{ }^{\circ} \mathrm{C}$ at $10^{\circ} \mathrm{C} \mathrm{min}^{-1}$ and then $80^{\circ} \mathrm{C}$ to $150{ }^{\circ} \mathrm{C}$ at $20^{\circ} \mathrm{C} \mathrm{min}{ }^{-1}$, flow rate at $1.0 \mathrm{~mL} \mathrm{~min}^{-1}$.

efficiency ${ }^{\mathbf{1 8}}$ of the TFTD column was obtained with the result of $88.8 \%$. The results suggested the high column efficiency and coating efficiency of the TFTD column, which are essential for a GC stationary phase to bring its separation performance into full play.

Polarity of a GC stationary phase is often evaluated by McReynolds constants that can be measured by five probe compounds, namely benzene $\left(\mathrm{X}^{\prime}\right)$, 1-butanol $\left(\mathrm{Y}^{\prime}\right)$, 2-pentanone $\left(\mathrm{Z}^{\prime}\right)$, 1-nitropropane $\left(\mathrm{U}^{\prime}\right)$ and pyridine $\left(\mathrm{S}^{\prime}\right)$. Accordingly, the determined McReynolds constants of the TFTD stationary phase at $120{ }^{\circ} \mathrm{C}$ are shown in Table 1 , which also provides the constants of the other two stationary phases for comparison.
Table 1 reveals that the TFTD stationary phase exhibits similar weak polarity to the TFT phase. In addition, the HP-5MS column of weak polarity was employed for comparison.

\subsection{Separation performance}

Separation performance and retention behaviors of the TFTD stationary phase were investigated by a number of mixtures composed of diverse types of analytes and isomers, and the results are presented in Fig. 3-6, respectively. Fig. 3 describes the separations of the mixture of seven benzene derivatives varying with alkyl, halogen, amino and hydroxyl substituents on the TFTD column in comparison to the TFT column.
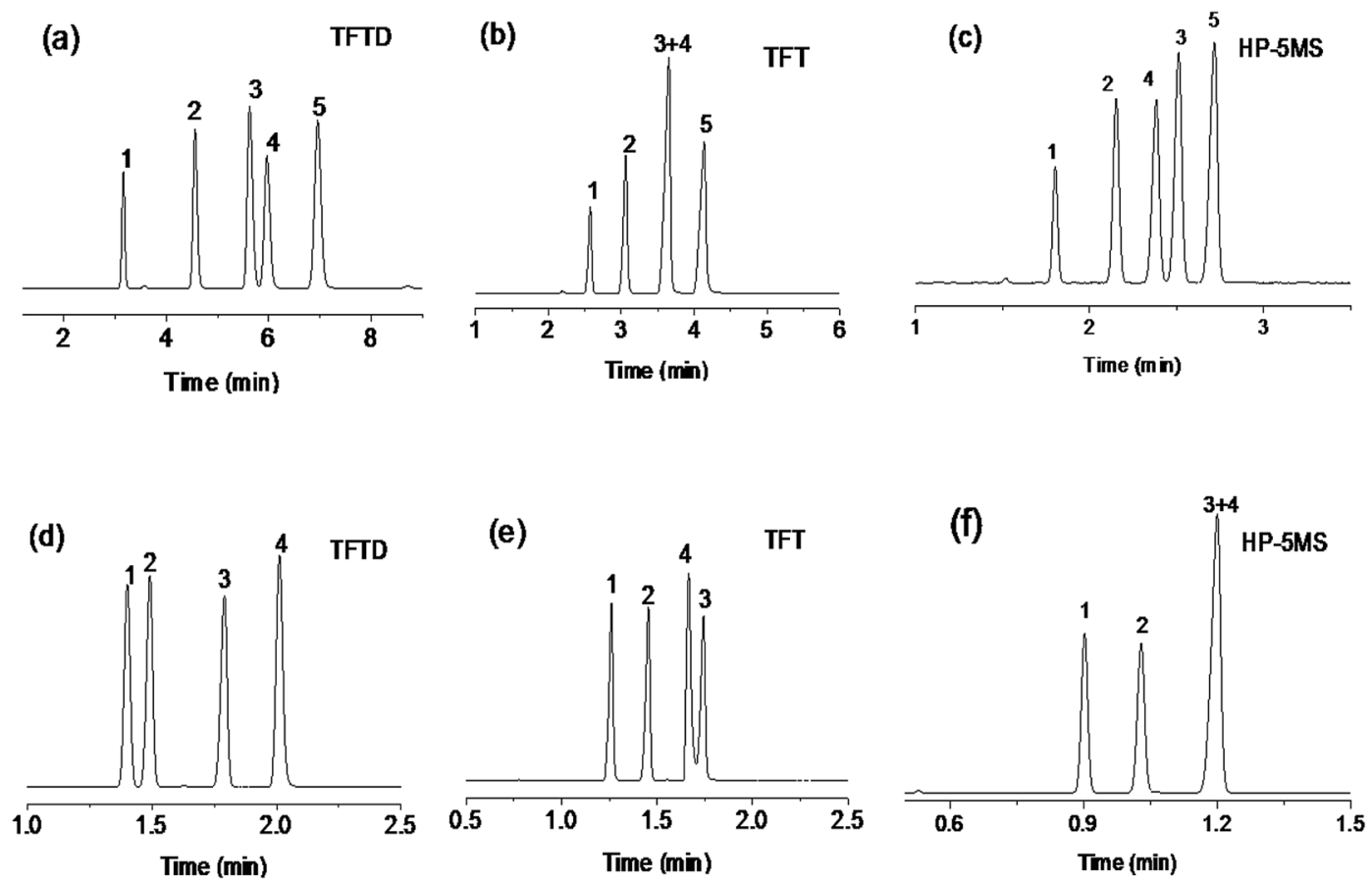

Fig. 4 GC separations of two isomer mixtures on the TFTD, TFT and HP-5MS columns. Peaks: in (a)-(c): (1) 2,6-dimethylphenol, (2) 2,4dimethylphenol, (3) 2,3-dimethylphenol, (4) 3,5-dimethylphenol, (5) 3,4-dimethylphenol; peaks in (d)-(f): (1) 2-chloroaniline, (2) 2-nitrotoluene, (3) 3-nitrotoluene, (4) 4-chloroaniline. GC conditions: isothermal at $100^{\circ} \mathrm{C}$, flow rate at $0.5 \mathrm{~mL} \mathrm{~min}-1$ for (a) $-(\mathrm{c}) ; 100{ }^{\circ} \mathrm{C}$ to $150{ }^{\circ} \mathrm{C}$ at $10^{\circ} \mathrm{C}$ min ${ }^{-1}$,

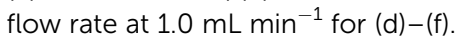



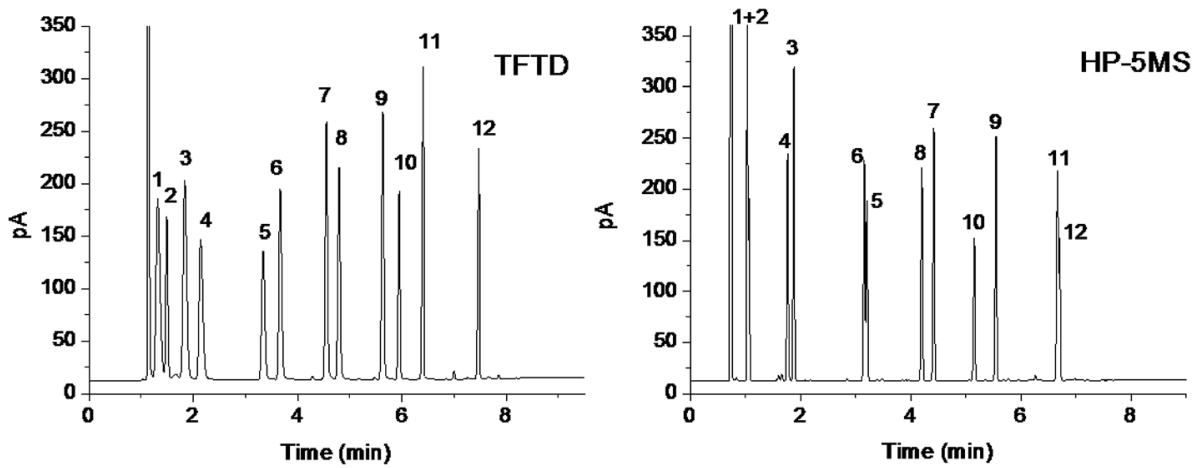

Fig. 5 GC separations of the mixture of 12 analytes on the TFTD column in comparison to the HP-5MS column. Peaks: (1) cyclohexane, (2) thiophene, (3) n-octane, (4) 1,3-dichloropropane, (5) 1-bromohexane, (6) bromobenzene, (7) n-butylbenzene, (8)1,2-dichlorobenzene, (9) 1,4diisopropylbenzene, (10) $1,3,5$-trichlorobenzene, (11) $n$-tridecane, (12) 1,6 -dibromohexane. GC conditions: $45^{\circ} \mathrm{C}$ for 1 min to $150{ }^{\circ} \mathrm{C}$ at

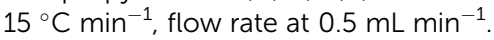

Apparently, the TFTD column baseline resolved all the analytes whereas the TFT column coeluted two pairs of them, i.e., $n$ butylbenzene/1,2-dichlorobenzene (peaks 2/3) and $n$ hexylbenzene/1,2,3-trichlorobenzene (peaks 5/6). Besides, the TFTD column distinctly prolong the retention of 4-fluoroaniline (peak 4) and 4-chlorophenol (peak 7). This phenomenon suggests the stronger interactions of the TFTD stationary phase with the benzene derivatives with amino, hydroxyl and/or halogen groups, possibly thanks to the cooperative effect of $\mathrm{H}$ bonding $(\mathrm{N}-\mathrm{H} \cdots \mathrm{S}, \mathrm{O}-\mathrm{H} \cdots \mathrm{S}),{ }^{19,20}$ halogen-bonding $(\mathrm{X} \cdots \mathrm{B}, \mathrm{B}=$ $\mathrm{N}, \mathrm{O}, \mathrm{S}$, aromatic $\pi$ system $)^{21,22}$ and $\pi-\pi$ electron-donor/ acceptor (EDA) interactions. As described previously, the TFTD stationary phase has a large $\pi$-conjugated system with much more thienylethynylene and thienyl units that outnumber the TFT phase. Additionally, the porous dendritic architecture provides more accessible interaction sites to the analytes, which
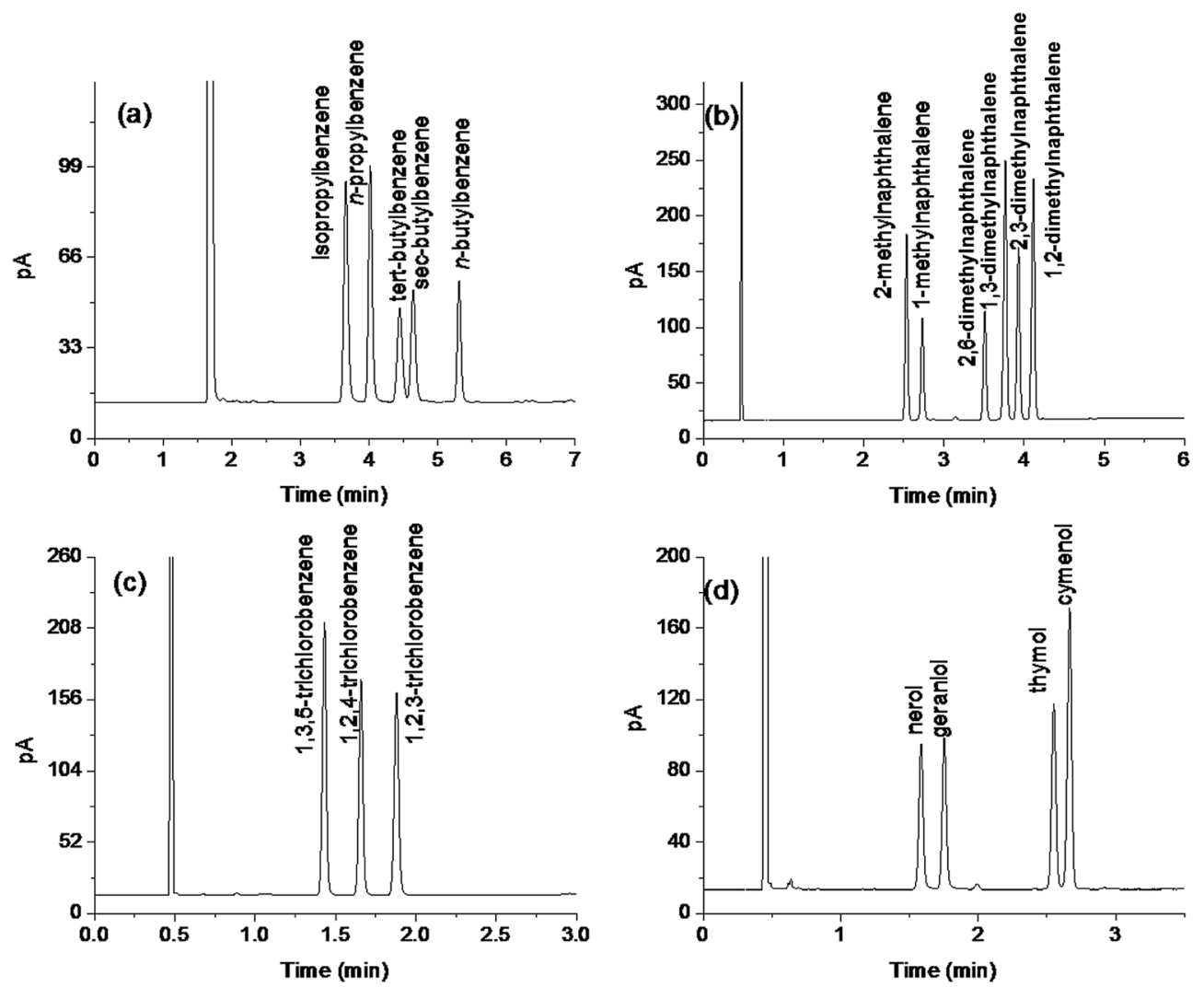

Fig. 6 Separations of (a) propylbenzene and butylbenzene isomers, (b) methylnaphthalene and dimethylnaphthalene isomers, (c) trichlorobenzene isomers and (d) nerol isomers and thymol isomers on the TFTD capillary column. GC conditions for (a): $50{ }^{\circ} \mathrm{C}$ to $150{ }^{\circ} \mathrm{C}$ at $10{ }^{\circ} \mathrm{C} \mathrm{min}-1$, flow rate at $0.35 \mathrm{~mL} \mathrm{~min}{ }^{-1}$; for (b)-(d), $100{ }^{\circ} \mathrm{C}$ to $150{ }^{\circ} \mathrm{C}$ at $10{ }^{\circ} \mathrm{C} \mathrm{min}^{-1}$, flow rate at $1 \mathrm{~mL} \mathrm{~min}^{-1}$. 
Table 2 Repeatability and reproducibility of TFTD columns on the retention times $\left(t_{R}, \min \right)$ of the analytes in the indicated mixture $(n=4)$

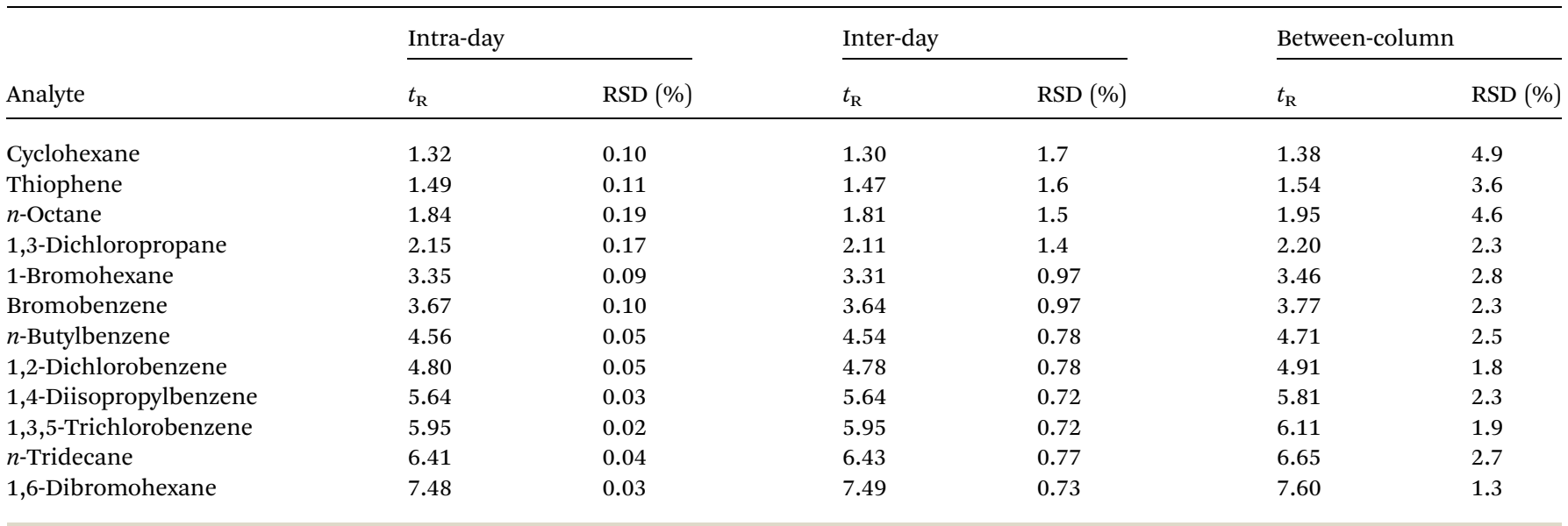

may further improve the resolution of 4-fluoroaniline and 4chlorophenol with their adjacent analytes. In contrast, the TFT phase retained shorter for the indicated analytes due to their weaker interactions described above.

The high resolving capability of the TFTD stationary phase motivated us to further explore its separation capability for aromatic isomers. Fig. 4a-c show the separations of dimethylphenol isomers on the TFTD, TFT and HP-5MS columns, respectively. Notably, the TFTD column achieved higher resolution of 2,3-dimethylphenol and 3,5-dimethylphenol (peaks 3/ $4, R=1.69)$ than the TFT column $(R=0)$. This may be achieved through the specific $\mathrm{H}$-bonding interactions $(\mathrm{O}-\mathrm{H} \cdots \mathrm{S})$ of the TFTD phase with the phenols. In addition, the TFTD column exhibited an elution reversal of the peaks $3 / 4$ in contrast to the HP-5MS column, resulting from the weaker H-bonding interaction of 2,3-dimethylphenol due to the steric hindrance of the neighboring methyl group. Fig. $4 \mathrm{~d}-\mathrm{f}$ describe the separations of nitrotoluenes and chloroanilines on the three columns. As can be observed, the TFTD column baseline separated all the analytes, proving its stronger resolving capability than the other columns. Particularly for 3-nitrotoluene and 4-chloroaniline (peaks 3/4) sharing the same boiling point (b.p.), they were not completely separated on the TFT column $(R=1.40)$ and totally overlapped on the HP-5MS column. In contrast, the TFTD stationary phase attained high resolution $(R=4.44)$ of them, stemming from its stronger H-bonding and halogen-bonding interactions with 4-chloroaniline (peak 4). This reason can also apply to their elution reversal on the TFTD column compared to the TFT column. The above results evidence the different retention behaviours of the TFTD stationary phase from the other two phases and also its advantageous resolving performance for phenols and anilines.

To further examine the separation capability of the TFTD stationary phase for other types of analytes, we employed a mixture of 12 analytes ranging from aliphatics to aromatics. The mixture was separated on the TFTD column in comparison to the HP-5MS column. As shown in Fig. 5, the TFTD column completely resolved all the analytes and outperformed the reference column that coeluted the pairs of cyclohexane/ thiophene (peaks 1/2), $n$-tridecane/1,6-dibromohexane (peaks 11/12) and mostly overlapped the pair of 1-bromohexane and bromobenzene (peak 5/6). The high resolving performance of the TFTD stationary phase for the indicated pairs may originate from its stronger $\pi-\pi$ interactions with thiophene and bromobenzene and its stronger halogen-bonding interaction with 1,6dibromohexane. Moreover, the TFTD stationary phase shows
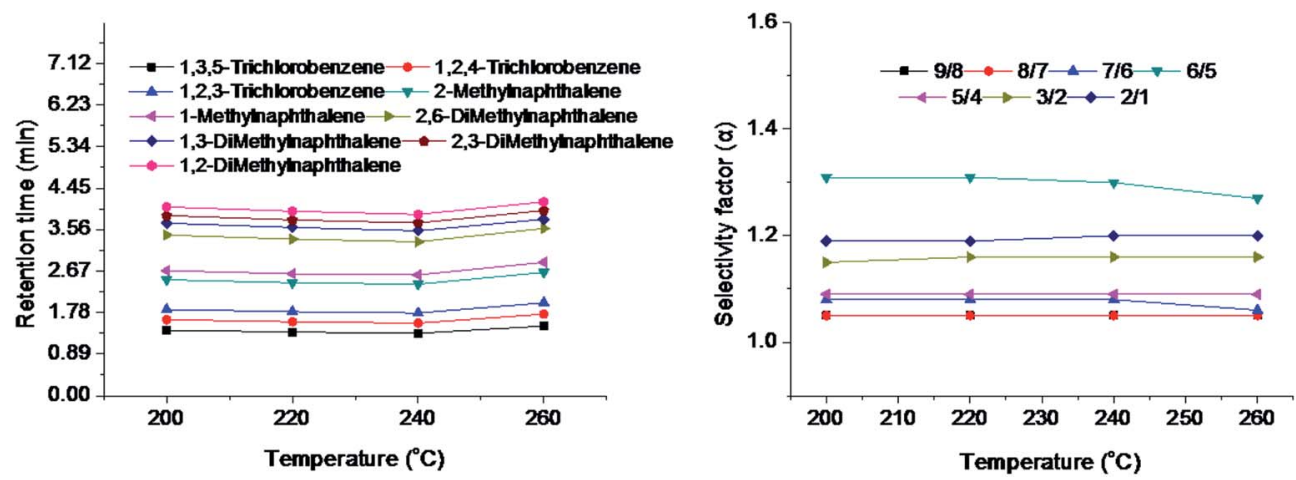

Fig. 7 Effect of conditioning temperatures on the retention times and selectivity factors of the isomers on the TFTD column. Analytes: (1) 1,3,5trichlorobenzene, (2) 1,2,4-trichlorobenzene, (3) 1,2,3-trichlorobenzene, (4) 2-methylnaphthalene, (5) 1-methylnaphthalene, (6) 2,6-dimethylnaphthalene, (7) 1,3-dimethylnaphthalene, (8) 2,3-dimethylnaphthalene, (9) 1,2-dimethyl-naphthalene. 
Table 3 Effect of column conditioning temperatures on the retention times ( $t_{\mathrm{R}}$, min) and selectivity factors $(\alpha)$ of the isomers on the TFTD column

\begin{tabular}{|c|c|c|c|c|c|c|c|c|c|c|}
\hline \multirow[b]{2}{*}{ Analyte } & \multicolumn{2}{|l|}{$200{ }^{\circ} \mathrm{C}$} & \multicolumn{2}{|c|}{$220^{\circ} \mathrm{C}$} & \multicolumn{2}{|c|}{$240^{\circ} \mathrm{C}$} & \multicolumn{2}{|c|}{$260{ }^{\circ} \mathrm{C}$} & \multicolumn{2}{|c|}{ RSD (\%) } \\
\hline & $t_{\mathrm{R}}$ & $\alpha$ & $t_{\mathrm{R}}$ & $\alpha$ & $t_{\mathrm{R}}$ & $\alpha$ & $t_{\mathrm{R}}$ & $\alpha$ & $t_{\mathrm{R}}$ & $\alpha$ \\
\hline 1,2,4-Trichlorobenzene & 1.626 & 1.19 & 1.582 & 1.19 & 1.554 & 1.20 & 1.746 & 1.20 & 5.2 & 0.69 \\
\hline 1,2,3-Trichlorobenzene & 1.844 & 1.15 & 1.797 & 1.16 & 1.770 & 1.16 & 1.988 & 1.16 & 5.3 & 0.43 \\
\hline 2-Methylnaphthalene & 2.482 & - & 2.418 & - & 2.389 & - & 2.643 & - & 4.2 & - \\
\hline 1,3-Dimethylnaphthalene & 3.669 & 1.08 & 3.608 & 1.08 & 3.535 & 1.08 & 3.784 & 1.06 & 3.0 & 0.93 \\
\hline 2,3-Dimethylnaphthalene & 3.862 & 1.05 & 3.771 & 1.05 & 3.705 & 1.05 & 3.972 & 1.05 & 3.0 & 0 \\
\hline 1,2-Dimethylnaphthalene & 4.047 & 1.05 & 3.957 & 1.05 & 3.889 & 1.05 & 4.157 & 1.05 & 2.9 & 0 \\
\hline
\end{tabular}

reversal elution for the middle four pairs of the analytes against their b.p. order. This reversal phenomenon further evidences the contribution of halogen-bonding, $\pi-\pi$ and $\pi-\pi$ EDA interactions to the later elution of 1,3-dichloropropane (peak 4), bromobenzene (peak 6), 1,2-dichlorobenzene (peak 8) and 1,3,5trichlorobenzene (peak 10).

Isomers are hard to be well separated because of their high resemblance in physicochemical properties. The TFTD column was investigated for its resolving capability for positional and structural isomers, including the mixtures of propylbenzenes/ butylbenzenes, methylnaphthalenes/dimethylnaphthalenes, trichlorobenzenes, and nerols/thymols. As shown in Fig. 6a, the TFTD column well separated the isomers of propylbenzenes and butylbenzenes and showed relatively longer retention for those with a linear alkyl group than those with a branched one. The longer retention of the former derives from its stronger $\pi-\pi$ interaction with the stationary phase thanks to its less hindrance and more close contact with the phase. Also, it achieved good resolution of the mixture of methylnaphthalenes and dimethylnaphthalenes (Fig. 6b). The later elution of 1methylnaphthalene than 2-methylnaphthalene can be attributed to its higher electron density and thus stronger $\pi-\pi$ interaction with the stationary phase..$^{23,24}$ The same reason also explained the later elution of 1,2-dimethylnaphthalene than 2,3dimethylnaphthalene against their b.p. order. Fig. $6 c$ shows the separation of the trichlorobenzene isomers. 1,2,3-
Trichlorobenzene eluted last owing to its relatively stronger halogen-bonding interaction via the adjacent three chlorine atoms. Moreover, the TFTD column also well separated the cis-/ trans-isomers of nerol and geraniol and the positional isomers of thymol and cymenol. The above results demonstrate the high-resolution performance of the TFTD stationary phase for diverse types of analytes and different retention behaviors from the TFT and polysiloxane stationary phases.

\subsection{Column repeatability and thermal stability}

The repeatability and reproducibility of the TFTD column were investigated by separations of the mixture of 12 analytes and evaluated by relative standard deviation (RSD\%) values on the retention times of the analytes in terms of intra-day, inter-day and between-column. As listed in Table 2, the obtained RSD values were in the range of $0.02-0.19 \%$ for intra-day, $0.72-1.7 \%$ for inter-day and $1.3-4.9 \%$ for between-column, respectively. These results proved its good column repeatability and reproducibility.

Column thermal stability was investigated by the isomer mixtures of methylnaphthalenes, dimethylnaphthalenes and trichlorobenzenes after the TFTD column was consecutively conditioned up to the temperatures of $200{ }^{\circ} \mathrm{C}, 220{ }^{\circ} \mathrm{C}, 240{ }^{\circ} \mathrm{C}$ and $260^{\circ} \mathrm{C}$ and held for $1 \mathrm{~h}$ at each of the high-end temperatures, respectively. Fig. 7 illustrates the effect of conditioning
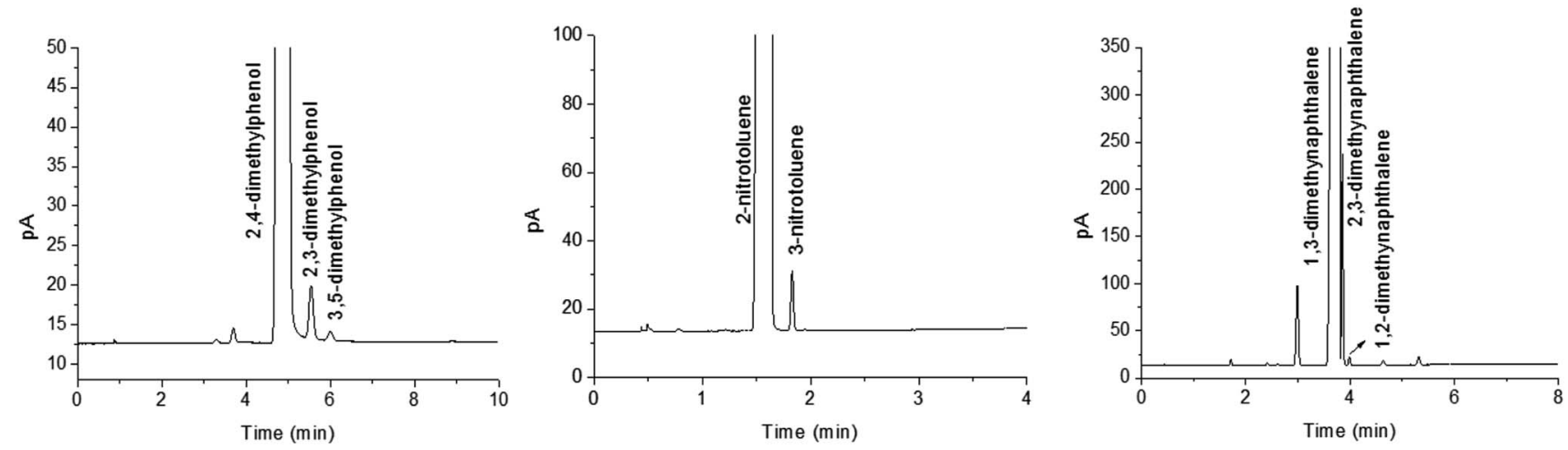

Fig. 8 Applications for the detection of isomer impurities in the commercial reagent samples on the TFTD column. 
Table 4 Results for the detection of isomer impurities in the commercial reagent samples on TFTD column

\begin{tabular}{|c|c|c|c|c|}
\hline Sample & Labeled purity (\%) & Measured purity (\%) & Impurity & Content (\%) \\
\hline \multirow[t]{2}{*}{ 2,4-Dimethylphenol } & \multirow[t]{2}{*}{$>95$} & \multirow[t]{2}{*}{99.4} & 2,3-Dimethylphenol & 0.41 \\
\hline & & & 3,5-Dimethylphenol & 0.08 \\
\hline \multirow[t]{2}{*}{ 1,3-Dimethynaphthalene } & \multirow[t]{2}{*}{$>95$} & \multirow[t]{2}{*}{96.1} & 2,3-Dimethynaphthalene & 2.11 \\
\hline & & & 1,2-Dimethynaphthalene & 0.10 \\
\hline
\end{tabular}

temperatures on both retention times and selectivity factors of the isomers on the column. Observably, after subjected to several cycles of the column aging, the TFTD column still maintained almost unchanged retention times and high selectivity for the isomers over the temperature range. The detailed data are also provided in Table 3, showing the RSD values of $2.9-5.3 \%$ for the retention times and less than $1.5 \%$ for the selectivity factors. The above results suggest its satisfying column thermal stability. Taking into consideration its separation performance and column lifetime, the TFTD column was recommended to be applied below $260{ }^{\circ} \mathrm{C}$.

\subsection{Applications for determination of isomer impurities in real samples}

In view of the excellent separation performance of the TFTD column for isomers, we applied the TFTD column for the detection of isomer impurities in commercial reagent samples. Fig. 8 provides the results for the determination of the reagent samples of 2,4-dimethylphenol, 2-nitrotoluene and 1,3-dimethynaphthalene, respectively. Table 4 summarizes their content results by peak area normalization method. As shown, the measured purity of the samples was in good agreement with their labeled purity, demonstrating the good potential of the TFTD column for practical applications in GC analysis.

\section{Conclusions}

This work presents the first example of exploring $\pi$-conjugated dendrimer materials as GC stationary phases. The TFTD stationary phase has a large $\pi$-conjugated dendritic architecture and favorable physicochemical features, and exhibits highresolution performance for a wide range of analytes, especially for positional isomers of phenols, anilines, alkylated and halogenated benzenes. Its high resolving capability mainly stems from the cooperative effect of its halogen-bonding and $\mathrm{H}^{-}$ bonding interactions through its sulfur-related units, which are barely reported in GC separations, in addition to $\pi-\pi$ stacking and $\pi-\pi$ EDA interactions. The present work demonstrates the feasibility of the TFTD stationary phase for GC separations and provides a research basis for exploring other dendrimer-based materials in this filed.

\section{Conflicts of interest}

There are no conflicts of interest to declare.

\section{Acknowledgements}

The authors are grateful for the financial support by the National Natural Science Foundation of China (21575013) and the 111 Project B07012 in China.

\section{References}

1 D. Astruc, E. Boisselier and C. Ornelas, Chem. Rev., 2010, 110, 1857-1959.

2 G. L. Schulz, M. Mastalerz, C. Q. Ma, M. Wienk, R. Janssen and P. Bäuerle, Macromolecules, 2013, 46, 2141-2151.

3 P. Kesharwani, K. Jain and N. K. Jain, Prog. Polym. Sci., 2014, 39, 268-307.

4 T. Kouketsu, S. H. Duan, T. Kai, S. Kazam and K. Yamada, J. Membr. Sci., 2007, 287, 51-59.

5 S. E. Sherman, Q. Xiao and V. Percec, Chem. Rev., 2017, 117, 6538-6631.

6 Q. M. Kainz and O. Reiser, Acc. Chem. Res., 2014, 47, 667-677.

7 M. Jaćkowska, S. Bocian and B. Buszewski, Analyst, 2012, 137, 4610-4617.

8 D. D. Guo, C. Y. Lou, P. M. Zhang, J. J. Zhang, N. N. Wang, S. C. Wu and Y. Zhu, J. Chromatogr. A, 2016, 1456, 113-122.

9 Y. Li, J. Yang, J. Jin, X. Sun, L. Wang and J. Chen, J. Chromatogr. A, 2014, 1337, 133-139.

10 J. J. Bao, C. H. Sun, Y. X. Li, R. J. Yuan, H. Xu, G. W. Chen and S. Y. Feng, Anal. Methods, 2015, 7, 6640-6646.

11 Y. Li, J. J. Yang, C. N. Huang, L. X. Wang, J. C. Wang and J. P. Chen, J. Chromatogr. A, 2015, 1392, 28-36.

12 I. Saeedi, P. Hashemi, Z. Ramezani and A. Badiei, Anal. Methods, 2015, 7, 10185-10191.

13 Y. M. Sun, K. Xiao, Y. Q. Liu, J. L. Wang, J. Pei, G. Yu and D. B. Zhu, Adv. Funct. Mater., 2005, 15, 818-822.

14 J. L. Wang, J. Luo, L. H. Liu, Q. F. Zhou, Y. Ma and J. Pei, Org. Lett., 2006, 8, 2281-2284.

15 J. L. Wang, J. Yan, Z. M. Tang, Q. Xiao, Y. G. Ma and J. Pei, J. Am. Chem. Soc., 2008, 130, 9952-9962.

16 F. Goubard and F. Dumur, RSC Adv., 2015, 5, 3521-3551. 17 Q. Lv, S. Feng, L. M. Jing, Q. Zhang, M. L. Qi, J. L. Wang, H. Bai and R. N. Fu, J. Chromatogr. A, 2016, 1454, 114-119.

18 E. F. Barry and R. L. Grob, Columns for gas chromatography: performance and selection, John Wiley \& Sons, New Jersey, 2007.

19 S. Bhattacharyya, A. Bhattacherjee, P. R. Shirhatti and S. Wategaonkar, J. Phys. Chem. A, 2013, 117, 8238-8250.

20 V. R. Mundlapati, S. Ghosh, A. Bhattacherjee, P. Tiwari and H. S. Biswal, J. Phys. Chem. Lett., 2015, 6, 1385-1389. 
21 L. C. Gilday, S. W. Robinson, T. A. Barendt, M. J. Langton, B. R. Mullaney and P. D. Beer, Chem. Rev., 2015, 115, 71187195.

22 G. Cavallo, P. Metrangolo, R. Milani, T. Pilati, A. Priimagi, G. Resnati and G. Terraneo, Chem. Rev., 2016, 116, 24782601.
23 P. George, C. W. Bock, J. J. Stezowski, T. Hildenbrand and J. P. Glusker, J. Phys. Chem., 1988, 92, 5656-5666.

24 L. Li, X. Y. Shi, R. J. Dai, Y. L. Deng, R. N. Fu and J. L. Gu, Chin. J. Anal. Chem., 2003, 31, 1322-1326. 